\title{
Effect of Oligomer Length on Vibrational Coupling and Energy Relaxation in Double- Stranded DNA
}

Gordon Hithell, ${ }^{1}$ Paul M. Donaldson, ${ }^{2}$ Gregory M. Greetham, ${ }^{2}$ Michael Towrie, ${ }^{2}$ Anthony W. Parker, ${ }^{2}$ Glenn A. Burley, ${ }^{3}$ Neil T. Hunt ${ }^{1 *}$

1) Department of Physics, University of Strathclyde, SUPA, 107 Rottenrow East, Glasgow, G4 ONG, UK

2) STFC Central Laser Facility, Research Complex at Harwell, Harwell Science and Innovation Campus, Didcot, OX11 OQX, UK

3) Department of Pure and Applied Chemistry, University of Strathclyde, 295 Cathedral Street, Glasgow, G1 1XL, UK Corresponding author, contact: neil.hunt@strath.ac.uk

\section{Abstract}

The effect of oligomer length on the vibrational mode coupling and energy relaxation mechanisms of AT-rich DNA oligomers in double- and single-stranded conformations has been investigated using two-dimensional infrared spectroscopy. Vibrational coupling of modes of the DNA bases to the symmetric stretching vibration of the backbone phosphate group was observed for oligomers long enough to form duplex-DNA structures. The coupling was lost upon melting of the duplex. No significant effect of oligomer length or DNA secondary structure was found on either the timescale for vibrational relaxation of the base modes or the mechanism, which was consistent with a cascade process from base modes to intermediate modes, some of which are located on the deoxyribose group, and subsequently to the phosphate backbone. The study shows that vibrational coupling between base and backbone requires formation of the double-helix structure while vibrational energy management is an inherent property of the nucleotide.

\section{Keywords}

Ultrafast spectroscopy; infrared; DNA; vibrational relaxation; vibrational coupling 


\section{Introduction}

The fundamental position occupied by deoxyribonucleic acid (DNA) within biology has motivated considerable efforts to understand both its structure and function in the solution phase. Although DNA consists of a polymer of just four repeating units, the deoxy-nucleotides of adenosine (A), guanosine (G), thymidine $(T)$ and cytidine $(C)$, the molecular physics of double stranded (ds)DNA is complex. The fact that dsDNA is biologically silent, acting only as a repository for genetic information, means that unravelling the dynamic behavior of DNA is as important as determining its static structure. In particular, the double-tosingle strand transition of DNA is a key step in the cellular machinery of transcription and replication. The role of the solvent either in defining the chemical properties of the helix or when participating in the intermolecular interactions of DNA, such as duplex formation or ligand binding, is also not properly understood.

Ultrafast infrared (IR) spectroscopy has been instrumental in revealing important dynamics of the DNA molecule. In the electronic ground state, IR pump-probe spectroscopy has been used to study the vibrational relaxation dynamics of base vibrational modes.[1] The application of two dimensional IR (2D-IR) spectroscopy has revealed the nature of vibrational modes of the bases and base-pairs, including the considerable changes brought about by Watson-Crick base pairing, which leads to delocalisation of base vibrations.[2-6] The couplings of these base modes makes them sensitive reporters of DNA structure and they have been used to determine the spectroscopic effects of base stacking $[7,8]$ as well as the sequence-dependent impact of ligand binding on the structure of the double helix.[9] Transient 2D-IR spectroscopy methods have observed the melting of dsDNA sequences.[10] The interaction of DNA with the aqueous solvent has also been studied with 2D-IR spectroscopy.[11] Focussing on the vibrational modes of the phosphodiester backbone, it has been shown that the primary contact of DNA with water is through the $\mathrm{PO}_{2}^{-}$unit.[11,12]

In recent publications, attempts have been made to understand how the individual components of the nucleotide (base, deoxyribose and phosphate backbone) combine to define the dynamics of the macromolecule. Studies of AT-rich DNA have shown a correlation between Watson-Crick base pairing and the creation of long-range phonon-type vibrational modes in the terahertz spectrum of DNA.[13,14] The use of two color-2D-IR spectroscopy has been used to probe dynamic links between the base modes and the sugar-phosphate backbone.[15] In a 15 base pair all-AT DNA sequence, the formation of the duplex was shown to lead to vibrational coupling of base carbonyl and ring-stretching vibrations with the symmetric stretching mode of the phosphate group. In addition, a long-range energy transfer mechanism was determined, featuring relaxation of the bases into a suite of intermediate modes coupled to the phosphate, some of which were located on the deoxyribose unit, prior to energy transfer to the phosphate group itself. This energy cascade is in good agreement with the recently proposed role of the phosphate unit as the primary point of contact with the solvent[11,12] and would seem to contribute to the fast energy dissipation mechanisms needed to protect DNA from photo-damage.[16-25] 
In this article, we explore these long-range dynamic phenomena of dsDNA further. By extending two-color $2 D-I R$ and vibrational relaxation experiments to a range of samples with varying oligomer lengths from one to 15 bases, we seek to determine whether the coupling observed between base and phosphate groups for the 15-base sequence is prevalent in other sequences and whether this interaction requires a minimum chain length to become established. In addition, we aim to discover whether the vibrational relaxation process is a property of the macromolecule or of the individual nucleotide.

\begin{tabular}{ccc}
\hline Oligomer & Sequence $\left(\mathbf{5}^{\prime}-\mathbf{3}^{\prime}\right)$ & Sample Concentration (mM) \\
\hline$n=15$ & ATTATTATTATATTA & 10 \\
$n=10$ & ATTATTATTA & 10 \\
$n=6$ & ATTATT & 20 \\
$n=4$ & AATT & 40 \\
$n=2$ & AT & 40 \\
$n=1$ & T & 300
\end{tabular}

Table 1. Summary of DNA oligomers studied. In the text, these are referred to using the $n$ value. For $n \geq 6$, all samples contained the oligomer shown and the complementary oligomer. For $n=2,4$ the oligomers are selfcomplementary. For $n=1$, stable base pairing in solution at room temperature was not possible and so this was used as a solution of the single monophosphate. The sample concentration for each oligomer is shown for reference.

a)

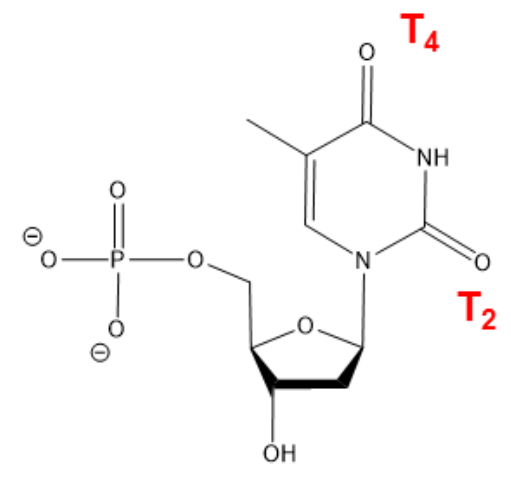

b)

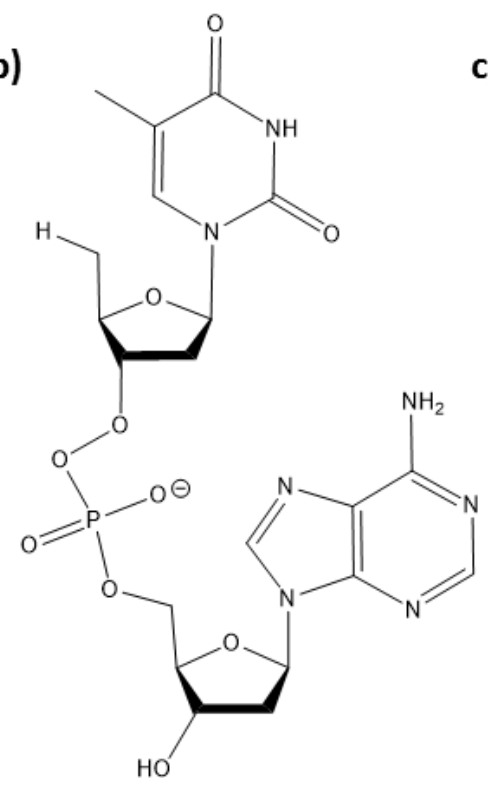

c)

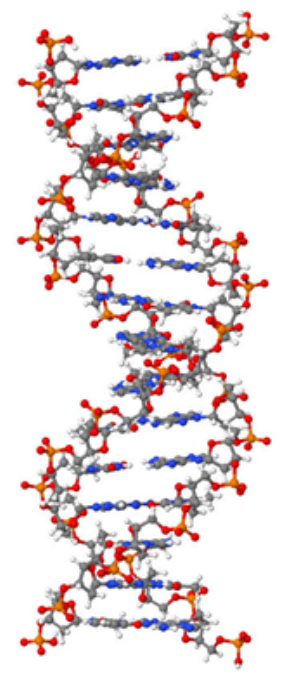

Figure 1. Schematic representation of the structures of some of the molecules studied. a) shows the structure of thymidine monophosphate $(n=1)$. The $T_{2}$ and $T_{4}$ carbonyl groups, referred to in the text, are highlighted. b) shows the AT dimer $(n=2)$ and c) shows the structure of the $n=15$ oligomer, also studied previously [14,15] (duplex image created in Jmol: an open - source Java viewer for chemical structures in 3D. http://www.jmol.org/.). 


\section{Experimental}

\section{Sample Preparation}

The DNA oligomers studied are shown in Table 1 with representative schematic structures in Figure 1. The oligomers were designed to encompass lengths from one to fifteen bases, while maintaining a sequence structure that was as similar as possible to the 15 base pair sequence studied previously (results for this sequence are also included in the data presented here for comparison).[15] Specifically, the ATT motif was a major component of the oligomers. This was chosen in order to minimise any slippage of base pairs or creation of unintended base pairing interactions. Henceforth, ' $n$ ' will refer to the number of bases within the specific DNA strand. For the oligomers that formed dsDNA structures at room temperature $(n=6,10,15$, see IR absorption results), mixtures of the oligomer with its complementary oligomer were used at all times and the sample concentrations quoted for these oligomers in Table 1 are the concentration of the resulting duplex. In the case of the oligomers with $n=2$ and 4, the oligomers used were self-complementary, but it was determined that dsDNA structures did not form at room temperature (see IR absorption results). Sample concentrations quoted for the $\mathrm{n}<4$ oligomers in Table 1 reflect oligomer concentrations. DNA oligomers along with their complementary oligomers were obtained from Eurofins Genomics $(n=6,10,15)$, Eurogentec ( $n=2$ and 4 ) and Sigma-Aldrich $(n=1)$. All samples were used without further purification. Oligomers were dissolved in a deuterated Tris buffer (100 mM Tris, $100 \mathrm{mM} \mathrm{NaCl}, \mathrm{pD7.4}$ ).

\section{IR Absorption and 2D-IR Spectroscopy}

For all IR spectroscopy measurements, the DNA sample was held between two $\mathrm{CaF}_{2}$ windows separated by a $25 \mu \mathrm{m}$-thick polytetrafluoroethylene spacer. The sample was housed in a thermostatically-controlled mount, allowing the temperature to be varied between 293 and $353 \mathrm{~K}$, accurate to $\pm 1 \mathrm{~K}$. FTIR spectra were recorded on a Bruker Vertex $70 \mathrm{FTIR}$ spectrometer with a resolution of $1 \mathrm{~cm}^{-1}$.

Ultrafast 2D-IR spectra were obtained using the LIFEtime spectrometer and the Fourier Transform 2D-IR method with the three input pulses arranged in a pseudo pump-probe geometry as described elsewhere.[15,26-28] For single color 2D-IR measurements, mid-IR laser pulses with a pulse duration of 300 fs were generated with central frequencies of $1650 \mathrm{~cm}^{-1}$ for use as pump and probe pulses for excitation and detection of the base modes of DNA. For two-color 2D-IR measurements, pulses with central frequencies of $1650 \mathrm{~cm}^{-1}$ were used to pump the base modes of DNA while probe pulses with a center frequency of 1090 $\mathrm{cm}^{-1}$ were used to detect the response of the DNA backbone modes. In both experiments, the pump pulse pairs were created using a mid-IR pulse shaper with phase cycling used to reduce any scattered light from the sample. The waiting time for the experiment was determined by the use of an optical delay stage. Parallel polarization of pump and probe pulses was used throughout. 

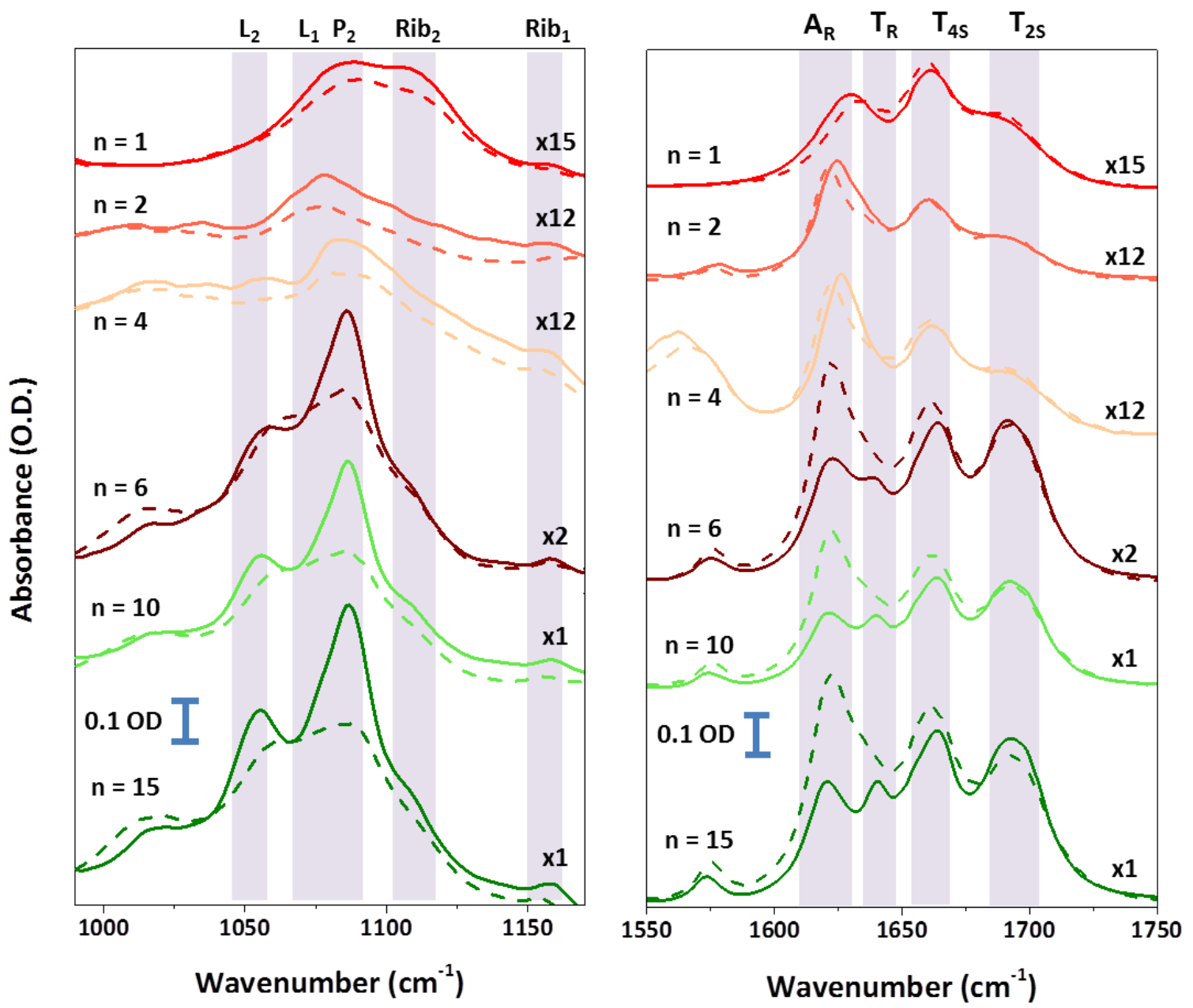

Figure 2. IR absorption spectra of the DNA samples $(n=1-15)$ in the backbone (left) and base (right) region of the IR spectrum. Solid lines indicate room temperature (293 K) spectra while the dashed lines show the result of heating to $353 \mathrm{~K}$. Grey shaded areas indicate the positions of particular vibrational modes described in the text along with the assignment of these modes using ssDNA notation. The absorbance scale is indicated by the blue scale bar. The scaling factor (e.g. x12) accompanying each spectrum gives an indication of relative spectral amplitudes for each oligomer. The factor quoted in each case reflects a combination of the sample concentration relative to that of the $n=15$ sequence (Table 1) and any amplitude scaling applied to the spectrum to enable comparison of all spectra on a single figure.

\section{Results}

\section{IR Absorption Spectroscopy - Base Region}

Figure 2 shows the IR absorption spectra of the DNA oligomers in both the DNA base $\left(1550-1750 \mathrm{~cm}^{-1}\right)$ and backbone (1000-1160 $\mathrm{cm}^{-1}$ ) spectral regions. The solid lines show the spectra at room temperature (293 K), while the dashed lines indicate the effect of increasing the temperature to $353 \mathrm{~K}$. 
Considering the base region spectra, for the oligomers with $n \geq 2$, three absorptions were observed at room temperature (Fig.2, solid lines) located near 1620, 1660 and $1690 \mathrm{~cm}^{-1}$. These are marked in the figure with grey shaded areas. In samples with a chain length $n \geq 6$, a fourth peak was resolved near $1640 \mathrm{~cm}^{-1}$. This peak was also present in the shorter $(n=2,4)$ oligomers but visible only as a shoulder on the $1620 \mathrm{~cm}^{-1} \mathrm{mode}$ Using designations of the modes appropriate for ssDNA [2-6,29] (see 2D-IR section below), these modes can be assigned to the $A_{R}$ ring stretching vibration of adenine $\left(1620 \mathrm{~cm}^{-1}\right)$ and three vibrational modes of thymine: a ring vibration $\left(T_{R}, 1640 \mathrm{~cm}^{-1}\right)$ and the $T_{4 S}$ and $T_{2 S}$ stretching vibrations of the $T_{4}$ and $T_{2}$ carbonyl groups (1660 and $1690 \mathrm{~cm}^{-1}$; see also Fig.1) respectively. These assignments are shown in Figure 2. For the $n=1$ sample, which contains only a single $T$ base, the $A_{R}$ mode was not present, so the peaks visible at 1690 and $1660 \mathrm{~cm}^{-}$ ${ }^{1}$ are assigned to the $T_{2 S}$ and $T_{4 S}$ modes while the third peak at $1630 \mathrm{~cm}^{-1}$ is assigned to the $T_{R}$ mode. [2] This peak is very sensitive to base pairing and stacking $[3-6,14,15]$ and so is shifted relative to that in the oligomers with $n>1$. All peak assignments are given in Table 2 .

\begin{tabular}{|c|c|c|c|c|c|c|c|c|c|}
\hline \multirow[t]{2}{*}{ Sample } & \multicolumn{5}{|c|}{ Backbone Region Assignments } & \multicolumn{4}{|c|}{ Base Region Assignments } \\
\hline & $\mathbf{L}_{2}$ & $\mathbf{L}_{1}$ & $\mathbf{P}_{2}$ & $\mathrm{Rib}_{2}$ & Rib $_{1}$ & $\left.A_{R} T / A_{r}^{a}\right)$ & $A T_{R} / T_{R}^{a)}$ & $A T_{4 S} / T_{4 s^{a}}$ & $A T_{2 S} / T_{2 S}{ }^{a)}$ \\
\hline $\mathrm{n}=15$ & 1055 & 1075 & 1087 & 1108 & 1158 & 1622 & $1640 / 1633$ & $1663 / 1661$ & 1693 \\
\hline $\mathrm{n}=10$ & 1055 & 1075 & 1085 & 1108 & 1158 & 1622 & $1640 / 1633$ & $1663 / 1661$ & 1693 \\
\hline $\mathrm{n}=6$ & 1056 & 1077 & 1085 & 1108 & 1158 & 1622 & $1639 / 1633$ & $1663 / 1661$ & 1692 \\
\hline$n=4$ & 1057 & 1077 & 1085 & 1108 & 1158 & $1626 / 1621$ & 1634 & $1661 / 1660$ & 1689 \\
\hline $\mathrm{n}=2$ & - & - & 1078 & 1106 & 1156 & $1624 / 1621$ & 1633 & 1660 & 1690 \\
\hline $\mathrm{n}=1$ & - & - & 1086 & 1108 & 1156 & - & $1630 / 1633$ & 1661 & 1687 \\
\hline
\end{tabular}

Table 2. Assignments and mode frequencies for all DNA samples studied. a) Frequency shifts of base modes caused by the loss of base pairing at higher temperatures are shown using dsDNA/ssDNA mode designations (see text).

The differing oligomer lengths can be divided into two distinct groups in terms of their response to an increase in temperature. The longer oligomers $(n \geq 6)$ showed a marked increase in the intensity of the $A_{R}$ mode upon heating to $353 \mathrm{~K}$, though the $T_{R}$ mode also shifted to lower frequency (Fig.2 dashed lines). By contrast, the spectrum of the shorter oligomers $(n \leq 4)$ at $353 \mathrm{~K}$ was largely the same as that at $293 \mathrm{~K}$. The 
most visible effect of increased temperature on the shorter oligomers was a minor shifting of the frequency of the $A_{R}$ mode.

The increase in amplitude of the $A_{R}$ mode at higher temperatures, as observed for the longer strands $(n \geq 6)$ can arise from the loss of base stacking upon melting of dsDNA strands.[29] To confirm that this was the case here, IR absorption spectra were recorded over a range of temperatures from 293 to $353 \mathrm{~K}$. The results of these experiments are shown in detail in the Supporting Information (Fig.S1), but briefly, the spectra of oligomers with $n \geq 6$ underwent temperature-dependent changes that were shown by Principal Component Analysis (PCA)[14] to follow a sigmoidal temperature dependence, with mid points of $329 \pm 2,313 \pm 2$ and $298 \pm 2 \mathrm{~K}$ for $n=15,10$ and 6 respectively. In the case of the oligomers with $n \leq 4$, a linear temperature dependence of the spectra was recovered, consistent with heating of the DNA oligomer but with no accompanying change in secondary structure. The spectral contributions to the temperature-dependent PCs of the long and short oligomers are shown in Fig.S1. The longer oligomers that displayed a sigmoidal temperature dependence $(n \geq 6)$ also showed a dramatic change in the $A_{R}$ peak amplitude and a smaller reduction in the $T_{2 s}$ mode amplitude, both of which are characteristic changes associated with duplex melting.[29] For the shorter oligomers, the PCA analysis showed that only a shift of the $A_{R}$ mode was correlated with increasing the temperature, consistent with observations from Figure 2 . Thus, we conclude that oligomers with $n \geq 6$ form a ds-DNA structure at room temperature, but oligomers with $n \leq 4$ do not. This was found to be consistent with traditional DNA melting curves obtained with UV-Visible absorption spectroscopy for the $n \geq 6$ sequences, the results of which are shown in the supporting information (Fig S2.)

\section{IR Absorption Spectroscopy - Backbone Region}

In the backbone region of the IR absorption spectrum (Fig.2; 1000-1160 $\mathrm{cm}^{-1}$ ), the spectra also fall into two groups depending on oligomer length; those with $n \geq 6$ and those with $n \leq 4$. For the longer oligomers $(n \geq$ 6), four features of interest were identifiable near 1050, 1085, 1105 and $1155 \mathrm{~cm}^{-1}$. These are marked with grey shaded areas in Figure 2 and can be assigned via previous studies[11] to the $L_{2}$ phosphodiester stretching mode, the $\mathrm{P}_{2}$ symmetric $\mathrm{PO}_{2}^{-}$stretch, and two modes located principally on the deoxyribose moiety, which we identify as $\mathrm{Rib}_{2}$ (which appears as a shoulder on the high frequency side of the $\mathrm{P}_{2}$ band) and Rib ${ }_{1}$, respectively. A further phosphodiester mode, designated $L_{1}$ is present as a low frequency shoulder on the $P_{2}$ mode, but is not clearly visible in the spectra as shown. Table 2 summarizes the assignments of the modes. For the samples with $n \geq 6$, the $\mathrm{P}_{2}$ mode decreased markedly in amplitude upon heating from 293 to $353 \mathrm{~K}$. This too followed a sigmoidal process (Fig.S3) and we attribute the effect to dsDNA melting.

For the oligomers with $n \leq 4$, the spectra were weaker and less well-defined in the backbone region, however, the $P_{2}$ mode and the two $\operatorname{Rib}_{x}$ modes $(x=1,2)$ were visible and lay close to the frequencies of the same modes in the longer strands. The backbone region spectra of the oligomers with $n \leq 4$ were largely temperature independent, consistent with the results observed in the base region (see also Fig.S3). 


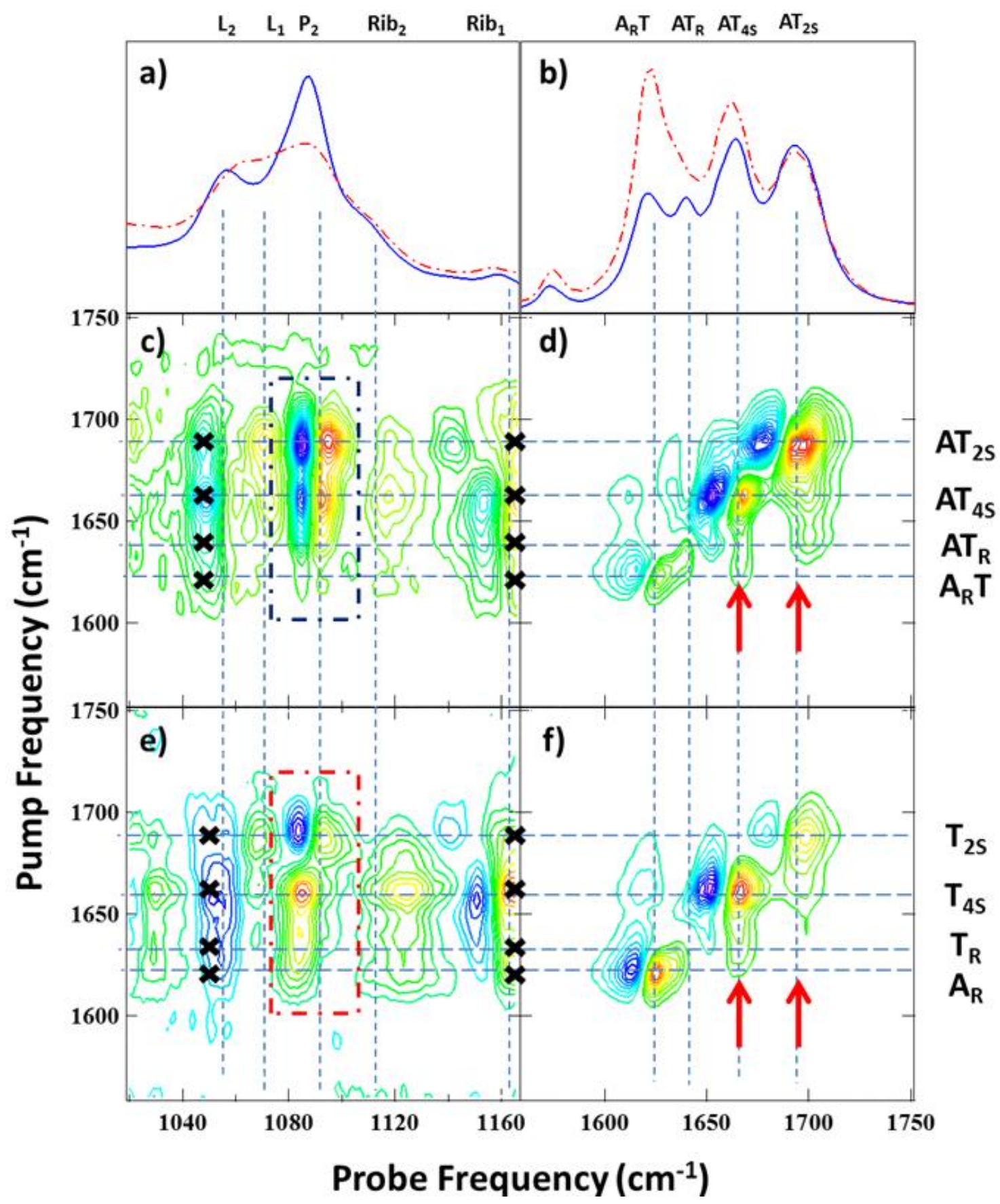

Figure 3. IR and 2D-IR spectra for $n=10$ oligomer: $a$ ) and b) $I R$ absorption spectra of the $n=10$ oligomer at $293 \mathrm{~K}$ (solid blue lines) and $353 \mathrm{~K}$ (dashed red lines) in the backbone (a) and base (b) region of the spectrum. c) and d) $2 D$-IR spectra of the $n=10$ oligomer at $293 \mathrm{~K} . c$ ) shows the two-color $2 D$-IR spectrum using excitation of the base modes and detection of the backbone modes. d) shows the one-color 2D-IR spectrum with excitation and detection in the base region of the spectrum. e) and f) $2 D-I R$ spectra of the $n=10$ oligomer at $353 \mathrm{~K}$ e) shows the two-color 2D-IR spectrum using excitation of the base modes and detection of the backbone modes. f) shows the one-color 2D-IR spectrum with excitation and detection in the base region of the spectrum. In all spectra, the waiting time was $400 \mathrm{fs}$ and relative pump-probe polarization was parallel. The color scale runs from red (negative) to blue (positive) with contour lines placed at $5 \%$ intervals. Contours for d) and f) start at the $10 \%$ level to reduce the effect of small features and noise. 


\section{D-IR Spectroscopy - Base Region}

Single color 2D-IR spectra (pumping and probing the base-region vibrational modes) of the $n=10$ oligomer are shown in Figure 3 at both $293 \mathrm{~K}$ (Fig3.(d)) and $353 \mathrm{~K}$ (Fig.3(f)) alongside the corresponding IR absorption spectra for reference (Fig.3(b)). The waiting time used to acquire these spectra was $400 \mathrm{fs}$, which was chosen to avoid the effects of temporal overlap of the pulses in the 2D-IR experiment. The 2D-IR spectroscopy of AT rich systems in this spectral region has been reported elsewhere and so we will provide only a brief overview of the results, as they were entirely consistent with previous observations. [14] The spectra of the $n=10$ oligomer (Fig.3) are representative of those recovered for all systems with $n \geq 6$, i.e. those that are capable of forming a dsDNA structure at room temperature. At 293K, the spectrum showed four negative peaks along the diagonal, assignable to the $v=0-1$ transitions of the four peaks visible in the IR absorption spectrum obtained at $293 \mathrm{~K}$ (Fig.3(b), blue, solid line). The v=1-2 transitions of each of these modes was observed in the 2D-IR spectrum as a positive peak shifted $\sim 15-18 \mathrm{~cm}^{-1}$ to lower probe frequency relative to the $\mathrm{v}=0-1$ transition. In addition to these peaks, off-diagonal features were observed linking all four of the diagonal peaks. These are shown by dashed guidelines in the figure and are assigned to vibrational coupling of the modes. These include peaks linking the $A_{R}$ mode to each of the $T_{R}, T_{2 S}$ and $T_{4 S}$ modes (see red arrows in Fig.3(d)). This coupling of modes previously assigned to 'adenine' or 'thymine' modes reflects the strong vibrational interactions leading to delocalisation of the vibrational modes across the base pair caused by Watson-Crick base pairing and this is reflected by the modified mode assignments ( $A T_{2 S}, A T_{4 S}, A T_{R}$ and $A_{R} T$ ) that will be used for the ds-DNA vibrational modes henceforth (Fig.3, Table 2).[2-6]

Heating the dsDNA oligomers with $n \geq 6$ to $353 \mathrm{~K}$ led to the loss of the off-diagonal peaks linking the $A_{R} T$ mode to the $A T_{R}, A T_{2 S}$ and $A T_{4 S}$ modes (red arrows, Fig.3(f)). This reflects the loss of base pairing upon duplex melting. A shift of the $A T_{R}$ mode to lower frequency as it changes to the $T_{R}$ mode was also observed.[14] It should be noted that the apparent change in intensity of the $T_{2 s}$ diagonal feature in Fig.3(f) is not due to a change in signal size but rather to due to a contouring effect arising from the large increase of the $A_{R}$ ondiagonal peak.

In the case of the shorter strands $(n \leq 4)$, the base region 2D-IR spectra at both 293 and $353 \mathrm{~K}$ closely resembled that of the $n=10$ oligomer at $353 \mathrm{~K}$ (Fig.3(f)), which is indicative of the lack of base-pairing present in the oligomers with fewer bases. 


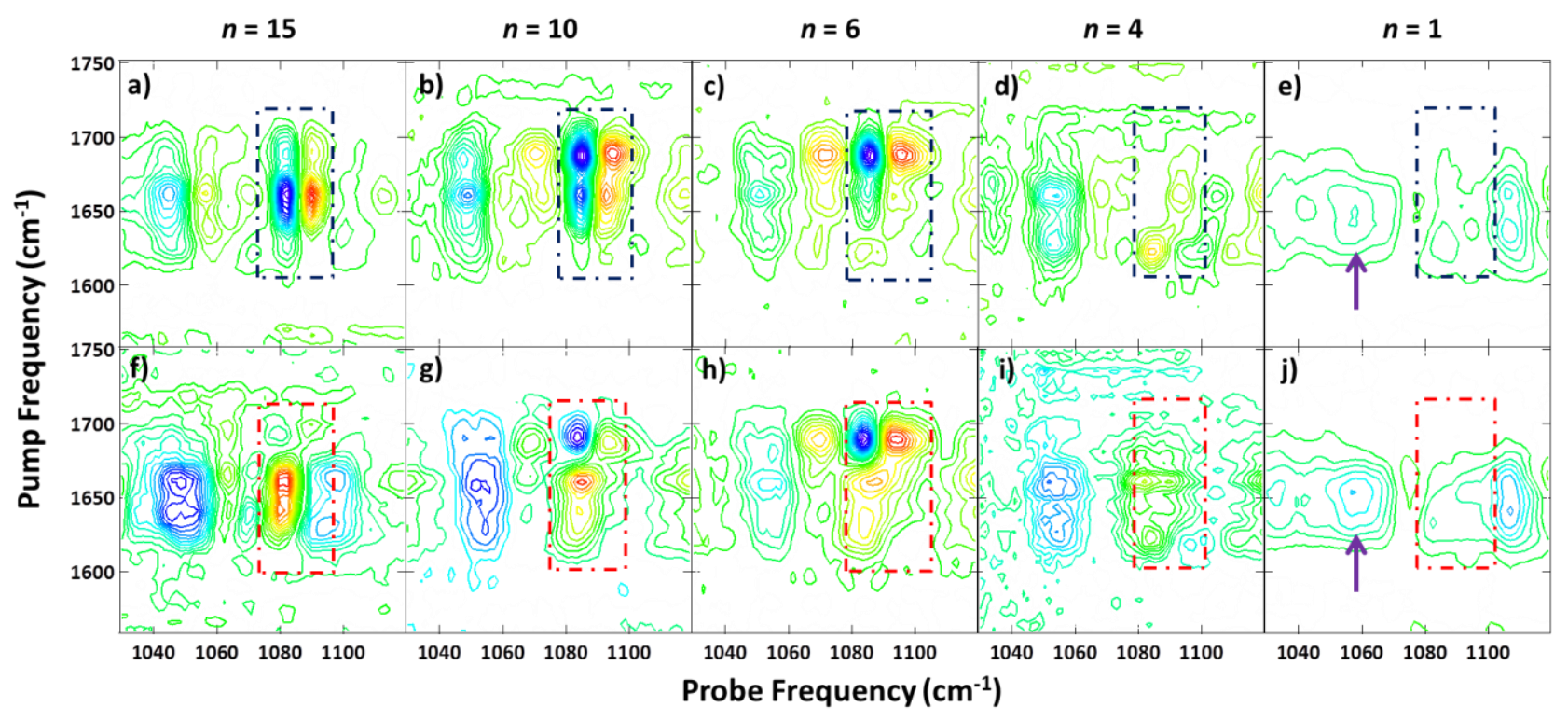

$293 \mathrm{~K}$

Figure 4. Two-color 2D-IR spectra of DNA samples as a function of oligomer length. a)-e) show the spectra at $293 \mathrm{~K}$ while f)-j) show the corresponding spectra at $353 \mathrm{~K}$. The blue (upper row) and red (lower row) boxes highlight the same regions of the spectrum highlighted in Figure 3 and show the regions of the spectra where temperature-dependent off-diagonal peaks linking base modes to the $P_{2}$ backbone mode appear for oligomers with $n \geq 6$. The color scale runs from red (negative) to blue (positive) with contour lines placed at $5 \%$ intervals.

\section{Backbone Region 2D-IR Spectra}

Two-color 2D-IR spectra of the 10-mer oligomer are shown in Fig.3(c) and Fig.3(e) at 293 and $353 \mathrm{~K}$ respectively. The waiting time used to acquire these spectra was $400 \mathrm{fs}$. In the two-color 2D-IR experiments, the pump frequency was set near $1650 \mathrm{~cm}^{-1}$ to excite the base vibrational modes discussed in the previous section while the probe frequency $\left(\sim 1100 \mathrm{~cm}^{-1}\right)$ interrogated the response of the backbone vibrations. Thus, the $2 D-I R$ spectra show the off-diagonal region of the $2 D-I R$ spectrum linking the base and backbone vibrational modes.

At $293 \mathrm{~K}$, the results for the $n=10$ oligomer (Fig.3(C)) showed a series of off-diagonal peaks linking the four base modes $\left(A T_{2 S}, A T_{4 S}, A T_{R}\right.$ and $\left.A_{R} T\right)$ with the $P_{2}$ symmetric stretching vibration of the phosphate group. These peaks are highlighted by the blue box in Fig.3(c). The peaks are identical to those reported for the $n=$ 15 oligomer previously.[15] Further, weaker off-diagonal features were also observed linking the base modes to the $L_{2}$ phosphodiester mode and to the Rib vibration near $1150 \mathrm{~cm}^{-1}$. These are highlighted by dashed lines in Fig.3(c).

Raising the temperature to $353 \mathrm{~K}$ caused a significant change in the two-color 2D-IR spectrum of the $n=10$ oligomer (Fig.3(e)). The strong peaks linking the base modes to the $\mathrm{P}_{2}$ vibration were lost for all except excitation of the $T_{2 S}$ mode (red box, Fig.3(e)). This resulted in off-diagonal modes between the $T_{4 S}, T_{R}$ and $A_{R}$ 
base vibrations and the $L_{1}$ phosphodiester mode becoming visible (red box, Fig.3(e)). The $L_{1}$ mode is located at $1075 \mathrm{~cm}^{-1}$ and appeared only as a shoulder to the $P_{2}$ band in the spectrum recorded at $293 \mathrm{~K}$ (Fig.3(c)). By contrast, the positions of off-diagonal peaks between the base modes and the $L_{2}$ and $\mathrm{Rib}_{1}$ modes were unchanged by increasing the temperature (see black crosses, Fig.3(c) and (e)), though some variations in amplitude were observed. The changes in the spectrum are assigned to changes in base to backbone mode coupling caused by melting of the helix, which is discussed in more detail below.

Considering the two-color 2D-IR spectra for all of the oligomers studied at both $293 \mathrm{~K}$ and $353 \mathrm{~K}$ (Fig.4), the temperature-dependent peak patterns identified in the $n=10$ spectra, shown by the blue and red boxes in Fig. 3(c,e), were replicated in the spectra for all oligomers with $n \geq 6$ (cf Fig.4(a-c) and Fig.4(f-h)). By contrast, the spectra for oligomers with $(n \leq 4)$ showed no evidence of the strong peaks linking base modes to the $\mathrm{P}_{2}$ vibration (Fig.4(d,e)) observed for the longer oligomers. Furthermore, the spectra of these shorter oligomers showed no particular variation with increased temperature (Fig.4(i,j). Thus we conclude that there is a correlation between the presence of these off-diagonal peaks linking base modes to the $P_{2}$ mode and the formation of the dsDNA structure.

The weaker peaks linking the base modes to the $L_{1}, L_{2}$ and Rib $_{1}$ modes were relatively unaffected by temperature and consistent across the spectra of all oligomers. It must however be considered that the $\mathrm{P}_{2}$ mode amplitude is greatly reduced in the spectra of the shorter oligomers $(n \leq 4)$ and reduced further with elevated sample temperature. Despite this, the fact that the $\mathrm{Rib}_{1}$ mode, although weaker than the $\mathrm{P}_{2}$ mode in every case (Fig.2), was visible in each spectrum (Fig.4(f-j) suggesting that this important off-diagonal peak is not falling below the level of detection.

\section{Vibrational Relaxation Dynamics - Base Relaxation Dynamics}

One-color 2D-IR spectra were obtained for each of the oligomers at a series of waiting times ranging from 0 fs to 5 ps (e.g. Fig.5(a)). As the waiting time increased, the amplitudes of the diagonal peaks were observed to decrease due to vibrational relaxation of the $v=1$ level. Plotting the peak amplitudes as a function of waiting time and fitting to a single exponential decay provided the vibrational relaxation time $\left(\mathrm{T}_{1}\right)$ of each of the base modes. A representative example of the vibrational relaxation of the diagonal features from the $n$ $=10$ oligomer is shown in Figure $5(b)$. In each case, the $T_{1}$ parameter was obtained from an average of the decay timescales of the $v=0-1$ and $v=1-2$ features, which both relate directly to the $v=1$ population. The results are shown in Table 3. Each of the base modes showed similar relaxation timescales, with a value of $\sim 660 \mathrm{fs}$ $\pm 50 \mathrm{fs}$ representing the bulk of the base modes at $293 \mathrm{~K}$. This is consistent with previous measurements for the $n=15$ oligomer, but slightly superior in terms of the spread of $T_{1}$ times recovered.[15] Little variation in the vibrational lifetimes of the modes was observed with changes in chain length (Table 3), though a generally slightly longer $T_{1}$ time was obtained for the $A_{R} T / A_{R}$ mode than for the higher frequency modes ( 760 fs \pm 50 fs). It is noted that the measurements were performed using ZZZZ polarisation, though no significant 
anisotropy has been observed on these timescales that would affect the outcome of the dynamics measured. Despite the fast $T_{1}$ times recovered by single exponential fitting, it is noted that a small residual signal is present at a waiting time of 5 ps (Fig.5). This is attributed to a small heating effect following relaxation of the DNA modes.
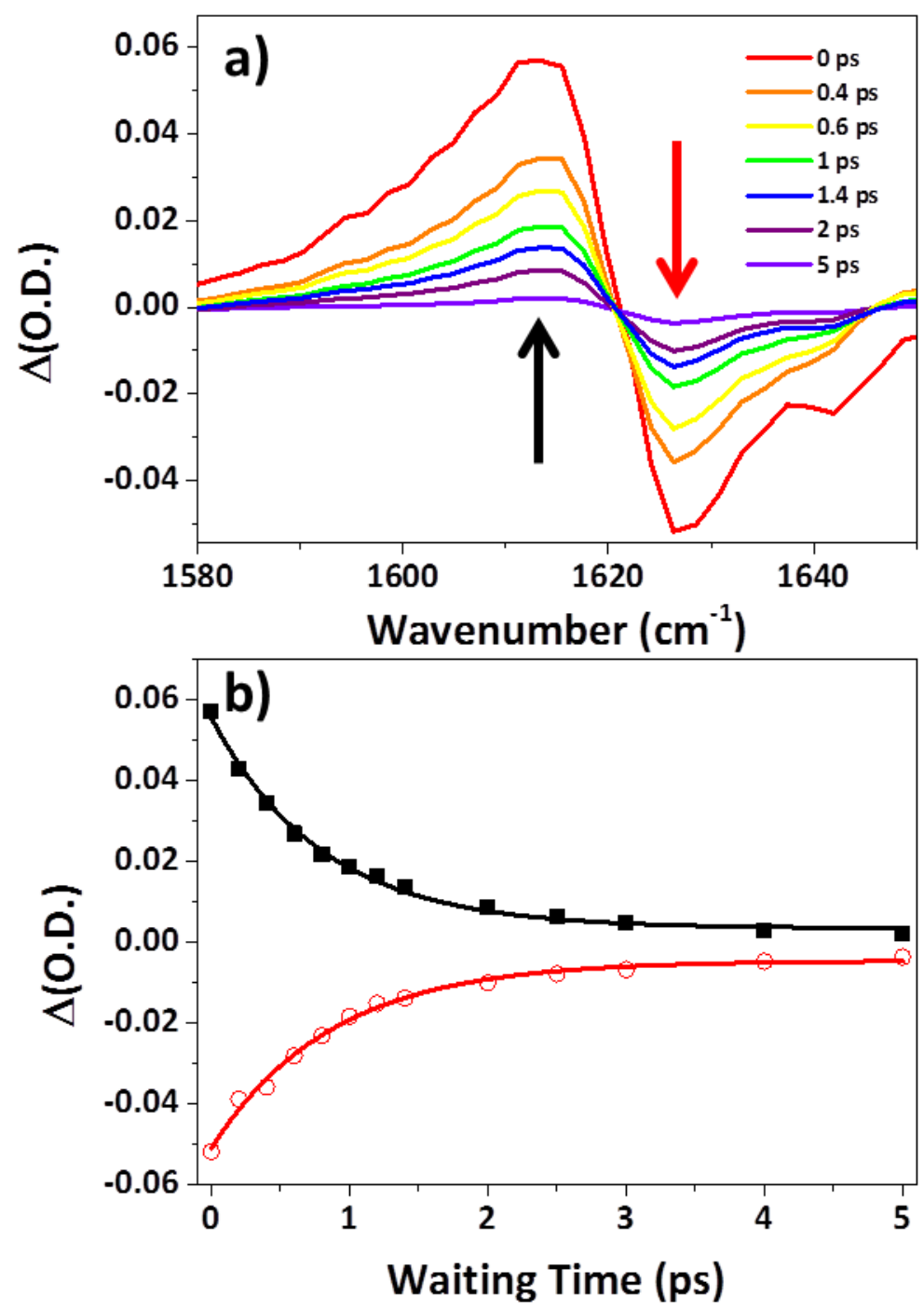

Figure 5. a) Cross sections through the $2 D$-IR spectrum of the $n=10$ oligomer at a pump frequency resonant with the $A_{R} T$ mode $\left(1622 \mathrm{~cm}^{-1}\right)$ showing the waiting time dependence of the diagonal peak observed. $b$ ) Temporal behavior of diagonal peak of the $A_{R} T$ mode in the one-color 2D-IR spectrum of the $n=10$ oligomer. Red open circles show the waiting time dependence of the amplitude of the $v=0-1$ transition (see red arrow in a), the black solid squares show the same for the $v=1-2$ transition (see black arrow in a). Solid lines show the results of fitting the data to single exponential decay function. 


\begin{tabular}{ccccc}
\hline Sample & $\mathbf{A}_{\mathrm{R}} \mathbf{T}(\mathbf{f s})$ & $\mathbf{A T}_{\mathrm{R}}(\mathbf{f s})$ & $\mathbf{A T}_{4 \mathrm{~S}}(\mathbf{f s})$ & $\mathbf{A T}_{2 \mathrm{~S}}$ (fs) \\
\hline $\mathrm{n}=15$ & $680 \pm 50$ & $520 \pm 70$ & $550 \pm 30$ & $580 \pm 30$ \\
$\mathrm{n}=10$ & $830 \pm 50$ & - & $560 \pm 20$ & $590 \pm 20$ \\
$\mathrm{n}=6$ & $710 \pm 30$ & $360 \pm 50$ & $570 \pm 30$ & $600 \pm 30$ \\
$\mathrm{n}=4$ & $710 \pm 40$ & - & $600 \pm 40$ & $630 \pm 10$ \\
$\mathrm{n}=2$ & $810 \pm 30$ & - & $650 \pm 40$ & $620 \pm 50$ \\
$\mathrm{n}=1$ & - & $530 \pm 50$ & $590 \pm 40$ & $640 \pm 30$
\end{tabular}

Table 3. Summary of the vibrational relaxation times $\left(T_{1}\right)$ obtained for base modes from the amplitudes of diagonal peaks appearing in one-color 2D-IR spectra recorded at a temperature of $293 \mathrm{~K}$.

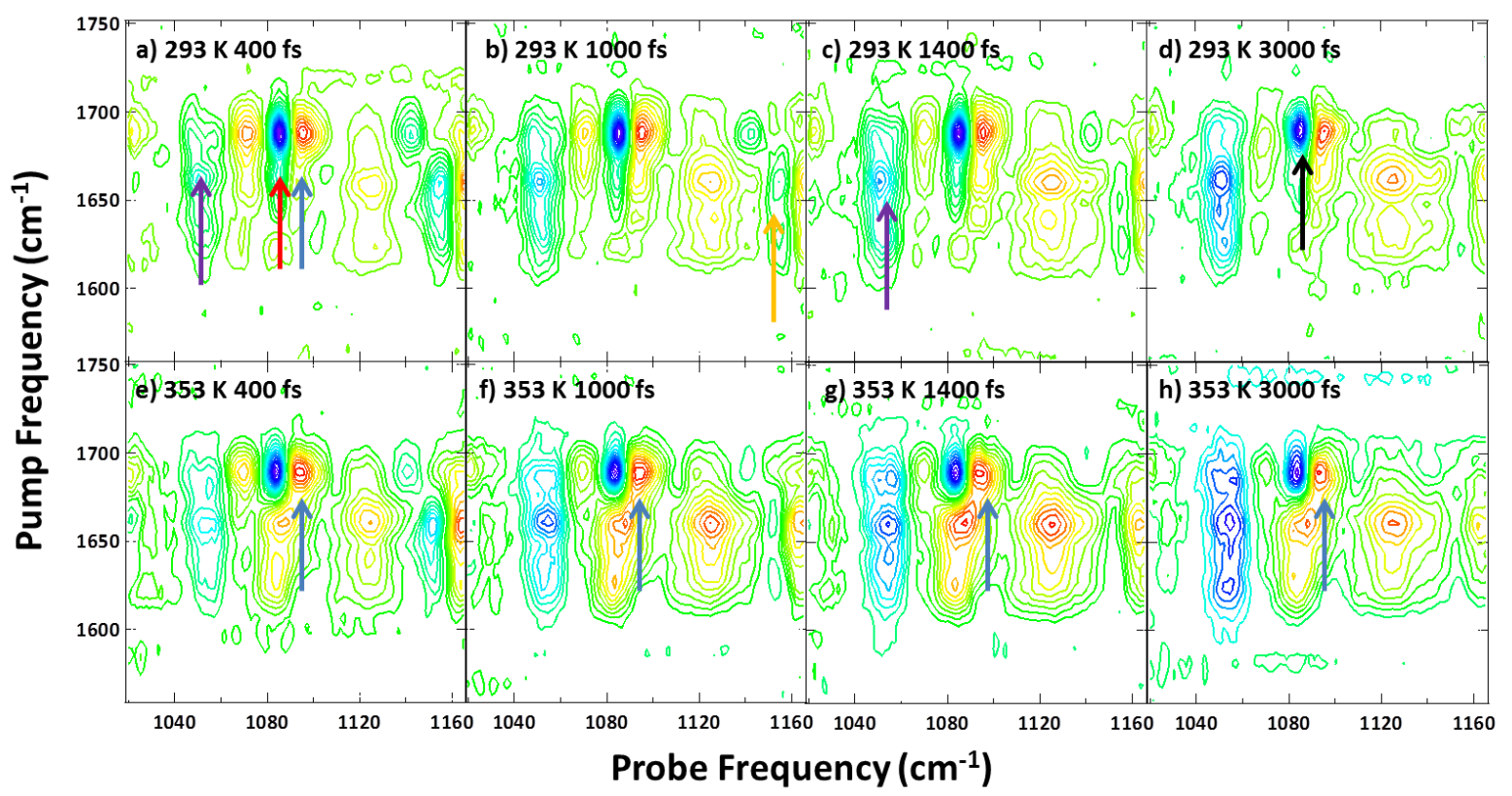

Figure 6. Waiting time-dependent two-color 2D-IR spectra of the $n=6$ oligomer. a)-d) show results at $293 \mathrm{~K}$, e)-h) show spectra for the corresponding waiting times but at $353 \mathrm{~K}$. Arrows identify peaks referred to in the text. Results are representative of all samples with $n \geq 6$. 


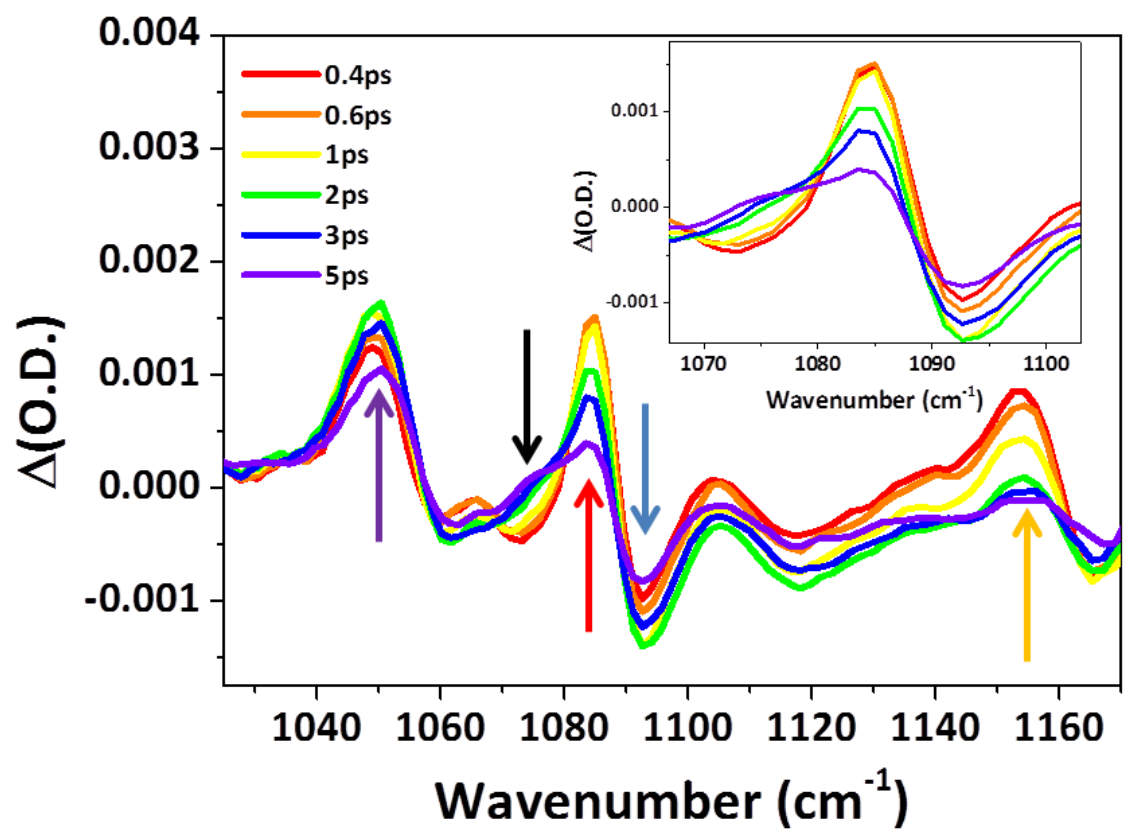

Figure 7. Waiting time dependent cross-sections through the two-color 2D-IR spectra of the $n=10$ sample at a pump frequency resonant with the $A T_{4 s}$ base mode $\left(1663 \mathrm{~cm}^{-1}\right)$, at $293 \mathrm{~K}$. Results are representative of all samples with $n \geq 6$ at $293 \mathrm{~K}$. The line colors run from red (waiting time $=0 \mathrm{ps}$ ) to purple (5ps). Arrows identify peaks referred to in the text and correspond to arrows of the same color in Figure 6. Inset shows expanded region of the spectrum near $1085 \mathrm{~cm}^{-1}$ ( $P_{2}$ mode).

\section{Backbone Relaxation Dynamics}

By recording two-color 2D-IR spectra as a function of waiting time from $0 \mathrm{fs}$ to $5 \mathrm{ps}$, it was possible to determine the vibrational relaxation mechanisms occurring to transfer vibrational energy from the base modes to the backbone modes. The results for the $n=15$ oligomer have been published previously and we seek here to extend the observations to shorter chain lengths.[15]

Phosphate group $\left(P_{2}\right)$ : As the off-diagonal peaks linking base vibrational modes to the $\mathrm{P}_{2}$ mode are the only ones that are structure- (i.e. temperature-) dependent, we begin by considering the dynamics of the offdiagonal peaks linking the base modes $A T_{2 S}, A T_{4 S}, A T_{R}$ and $A_{R} T$ to the $P_{2}$ stretching vibration in dsDNA oligomers ( $n \geq 6$ at $293 \mathrm{~K}$ ). We note that these peaks do not appear in the two-color 2D-IR spectra of the oligomers with $n \leq 4$ (Fig.4). A series of waiting-time dependent two-color 2D-IR spectra of the $n=6$ oligomer are shown in Figure 6 while cross-sections through the two-color 2D-IR spectrum of the $n=10$ oligomer are shown at a pump-frequency of $1660 \mathrm{~cm}^{-1}$, resonant with the $A T_{45}$ mode, at a range of waiting times (Fig.7). The data in these figures are both representative of the consistent spectra obtained for the longer oligomers $(n \geq 6)$ at room temperature, irrespective of oligomer length and the base mode excited. 
The cross-sections show that the off-diagonal peak linking base modes to the $P_{2}$ mode has three contributions. A negative peak (Fig.6(a) and Fig.7, blue arrow) was accompanied at short waiting times ( 400 fs) by a narrow, positive feature shifted by around $7 \mathrm{~cm}^{-1}$ to lower probe frequencies (Fig.6(a) and Fig.7, red arrow). As the waiting time increased, the negative peak grew in amplitude and then decayed, while the narrow positive feature decreased in amplitude, to be replaced by a broader peak shifted by around $12 \mathrm{~cm}^{-1}$ to lower probe frequencies relative to the negative peak (Fig.6(a) and Fig.7, black arrow).
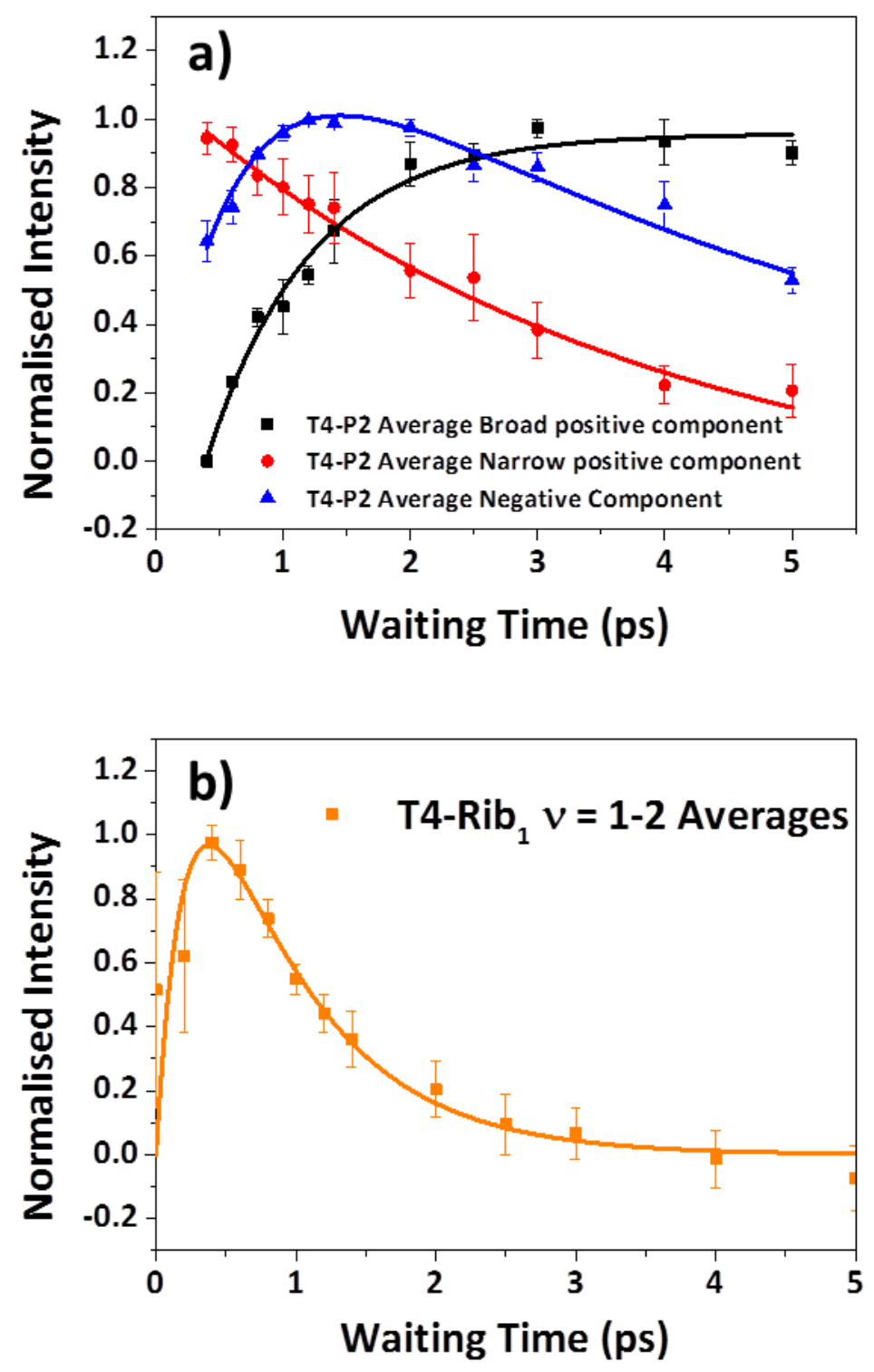

Figure 8. a) Waiting time dependence of the amplitudes of off-diagonal peaks in two color-2D-IR spectra linking base vibrational modes to the $P_{2}$ mode. Data shows the average response for all dsDNA samples $(n \geq$ 6) at $293 \mathrm{~K}$. The error bars show the spread of values recovered across the three samples. Blue, red and black traces correspond to the peaks identified by arrows of the same color in Figs.6 and 7. b) Time dependence of the off-diagonal peaks in two color-2D-IR spectra linking base modes to the Rib ${ }_{1}$ vibrational mode. Data shows the average response for all dsDNA samples at $293 \mathrm{~K}$. The error bars show the spread of values recovered across all samples. 
The time-dependent dynamics of the three components of the $\mathrm{P}_{2}$ off-diagonal peaks were obtained from the two-color 2D-IR data by comparing peak amplitudes as a function of waiting time (293 K). The results are shown in Figure 8(a). As the results recovered for all oligomers with $n \geq 6$ were similar, Fig.8(a) reproduces the average response of the three peaks (one negative and two positive features) across the three oligomers $(n=6,10,15)$ and the error bars show the spread of the data across the three oligomers. The dynamics were well-represented by exponential functions in all cases. In the case of the negative peak (Fig.8(a), blue and blue arrow Fig.7), an average rise time of $500 \pm 150 \mathrm{fs}$ and a decay of $\sim 5000 \pm 2000 \mathrm{fs}$ was observed. It is noted that the accessible time-range of the data, as defined by the $T_{1}$ time of the base modes, prevented accurate definition of the long-time relaxation dynamics. The red-shifted positive feature (Fig.8(a), black, and black arrow Fig.7) showed a similar rise time and a long-lived decay (>5000 fs). In the case of the sharper positive peak (Fig.8(a), red and red arrow Fig.7), this showed dynamics consistent with an exponential decay ( $\sim 000 \mathrm{fs})$. These results are summarised in Table S1.

The complex spectroscopy observed for the dsDNA oligomers with $n=10$ and 6 is identical to that reported for the $n=15$ oligomer previously[15] and is assigned to a combination of vibrational coupling and energy transfer resulting from excitation of the base modes. The negative peak and sharp positive feature present at short waiting times are due to the effects of vibrational coupling of the base and $\mathrm{P}_{2}$ modes caused by the formation of the double helix dsDNA structure. This explains the loss of the off-diagonal peaks to the $\mathrm{P}_{2}$ mode upon heating of the sample (Fig.4). In the case of coupling, the separation of the positive and negative features along the probe frequency axis is determined by the off-diagonal anharmonicity of the coupled modes.

The growth of the negative feature and appearance of the red-shifted positive feature at longer waiting times are attributable to energy transfer dynamics. The rise time of $500 \pm 150 \mathrm{fs}$ overlaps with that observed for relaxation of the base modes ( $650 \pm 50 \mathrm{fs}$ ) to within experimental accuracy, suggesting direct energy transfer from base to $\mathrm{PO}_{2}^{-}$group. However, in the case of energy transfer, the frequency shift of the positive feature from the negative peak is defined by the diagonal anharmonicity of the mode to which energy is transferred. This does not exactly match the known anharmonicity of the $P_{2}$ mode (12 vs $8 \mathrm{~cm}^{-1}[11]$ ) and so we conclude that the energy transfer from the base modes occurs to an intermediate mode (or modes), which couple to the $\mathrm{P}_{2}$ mode.[30,31] This leads to the rising component observed in the $\mathrm{P}_{2}$ off-diagonal peak. The decay timescale of these off-diagonal peaks is significantly longer than the relaxation $\left(T_{1}\right)$ time of the $P_{2}$ mode[11] and so we further conclude that we are observing the first step in a complex energy transfer pathway that ultimately leads to population of the $P_{2}$ mode within a few picoseconds. This is supported by the fact that the anharmonic shift of the positive feature assigned to energy transfer in the 2D-IR spectrum continues to evolve in terms of its position throughout the waiting time range studied (see inset Fig.7), consistent with continuous transfer of energy between a network of modes, which couple to $P_{2}$. The fact that 
the positive feature assigned to coupling also decays slowly, further suggests that some of the intermediate modes receiving energy from the bases are coupled to the $\mathrm{P}_{2}$ mode in the dsDNA configuration.

We now turn to the behavior of off-diagonal peaks linking base modes to the $P_{2}$ mode at elevated temperature $(353 \mathrm{~K})$, where all oligomers are in the ssDNA conformation. At $353 \mathrm{~K}$, the majority of the offdiagonal peaks linking the base modes to the $P_{2}$ mode were lost with the exception of that between the $T_{2 S}$ and $P_{2}$ modes for the oligomers with $n \geq 6$. The behavior of this feature can be seen in Figure $6(e-h)$ (blue arrow). In this case, the peak showed the rising ( $\sim 500 \mathrm{fs}$ ) and decaying ( $\sim 5000 \mathrm{fs})$ dynamics characteristic of the energy transfer mechanism observed in the dsDNA sample, but without the additional narrow positive lineshape component assignable to vibrational coupling that was specific to the dsDNA samples.

In the case of the oligomers with $n \leq 4$, the off-diagonal peak to the $P_{2}$ modes were not clearly visible and as such dynamics for these oligomers cannot be compared with those with $n \geq 6$. From the backbone region offdiagonal features present in the 2D-IR spectra for oligomers with $n \leq 4$ the evidence of an energy transfer mechanism is found through the dynamics recovered for the $L_{2}$ and $\operatorname{Rib}_{2}$ modes which we will now discuss.

Phosphodiester backbone $\left(L_{2}\right)$ : The off-diagonal peak linking the $A T_{45}$ base vibration to the $L_{2}$ phosphodiester stretching vibration is visible in Fig.6 and Fig.7 (purple arrows). This off-diagonal peak was visible in all samples at both low and high temperatures, with the exception of the TMP sample where the phosphodiester linkages are not present in the structure. It is noted that the expected dispersive lineshape is not clearly observed due to the presence of overlapping features assignable to the $L_{1}$ vibrational mode.[15] However, a peak is observed at $1070 \mathrm{~cm}^{-1}$ in the FTIR spectrum of the $n=1$ sample, which may have a similar origin. This is visible in the 2D-IR spectra (Fig.4(e,j), purple arrows). The temporal dynamics recovered for the $L_{2}$ offdiagonal peaks are shown in Fig.S4 and Table S1. In all samples, this mode showed dynamics consistent with the energy transfer process described for the $P_{2}$ mode, with a rise time of $730 \mathrm{fs} \pm 120 \mathrm{fs}$ and decay of $>5000$ fs being observed. This is slightly longer than observed for the $P_{2}$ mode but again the errors overlap preventing definitive separation. The striking result is the agreement across the chain lengths, as shown in the figure by the small error bars, further reinforcing the result obtained from the $P_{2}$ mode that the vibrational relaxation mechanism is relatively robust irrespective of the chain length and conformation (ds or ss) of the sample. This is different to the coupling phenomenon, which is unique to dsDNA.

Ribose (Rib $)_{1}$ ): The most intense off-diagonal peak linking the base modes to vibrational modes of the ribose group is located at $1155 \mathrm{~cm}^{-1}$, identified as Rib ${ }_{1}$. This off-diagonal is shown in Fig. 6 and Fig.7 (orange arrows). This peak was present in the spectra of all oligomers at both low and high temperatures. Interestingly, the dynamics recovered for the Rib 1 mode were different to those discussed for the $P_{2}$ and $L_{2}$ modes (Fig.8(b)). In the case of the Rib ${ }_{1}$ off-diagonal peak, a slightly faster rise (450 $\pm 40 \mathrm{fs}$ ) was followed by a significantly faster decay time (1200-1600 fs) than was observed for off-diagonal peaks to the $P_{2}$ and $L_{2}$ modes. Once again, the dynamics of this off-diagonal peak were robust across the samples studied, as can be seen by the 
very small spread in the data in Figure $8(\mathrm{~b})$. Furthermore, the relaxation timescales were invariant with increased temperature and so were not sensitive to the dsDNA to ssDNA transition.

It is noted that the rise time was a little too fast to allow definitive measurement given the time resolution of the LIFEtime instrument, the qualitative indication is that it is faster than that observed for the $P_{2}$ and $L_{2}$ backbone modes. It is impossible to rule-out absolutely a contribution from vibrational coupling from the base modes to the Rib 1 mode however, although if it is present then it must be conformation independent, as opposed to that seen for the $P_{2}$ mode. Similar dynamics were reported previously for the base-Rib $b_{2}$ mode of the $n=15$ oligomer and the faster rise and decay times were assigned to the Rib $\mathrm{B}_{2}$ mode playing an intermediate role in the energy transfer dynamics.[15] Thus, the data appears consistent with the ribose group acting as a link between the base and backbone dynamically as part of the energy transfer cascade in a manner that reflects its physical position within the DNA structure.

\section{Discussion}

It is clear from the data presented that creation of dsDNA structures, as seen in the oligomers with $n \geq 6$, leads to specific features in the off-diagonal region of the 2D-IR spectrum associated with coupling of base vibrational modes to the $\mathrm{P}_{2}$ symmetric stretching mode of the $\mathrm{PO}_{2}^{-}$unit. Upon melting of the DNA duplex these features were no longer present. An off-diagonal peak linking $T_{25}$ to $P_{2}$ remained at high temperature but lacked the spectroscopic signature of vibrational coupling, indicating only the presence of an energy transfer process between these modes at elevated temperature. The coupling of base modes to the $\mathrm{P}_{2}$ mode has been previously proposed to arise from the conformation of the helix bringing the transition dipole moments of the $\mathrm{PO}_{2}^{-}$modes and base modes into common alignment perpendicular to the helix direction.[15] It has been suggested that this alignment may also result in the increased $\mathrm{P}_{2}$ amplitude in dsDNA samples (Fig.2).[15] The commonality of the feature across the duplex-forming oligomers studied here lends further support to this, but further simulations are required to fully understand this behavior.

The shorter oligomers $(n \leq 4)$ used in this study do not show the same coupling behavior as the longer ones. In the case of the $n=4$ oligomer there is some indication of interaction between the $T_{4 s}$ mode and the $P_{2}$ mode (Fig.4(d)) however the amplitude of this feature is very small compared to other features in the spectrum. Upon heating of the sample, this feature was also lost as observed in the longer oligomers. This points to some change in the conformation in the $n=4$ oligomer upon heating, despite the IR absorption and base region 2D-IR spectra indicating that the $n=4$ oligomer can be considered to be ss-DNA at all temperatures. As the number of bases required for a full turn in a double helix is ten,[32] there is some possibility of weak base pairing between the $n=4$ oligomers, which would be disrupted at higher temperatures. The indications from the 2D-IR data are that, at room temperature, this is insufficient to form the vibrational coupling interactions observed in the longer oligomers however and this may be due to the 
fact that a half-turn of the DNA helix is not achievable. In the $n=1$ spectrum, there are no features coupling the base modes to the $\mathrm{P}_{2}$ mode, indicating firmly that these peaks are a feature of the double helix as opposed to a nucleotide-based mechanism.

Although the energy transfer processes were remarkably similar for oligomer lengths studied, a notable difference between the shorter and longer oligomers was observed in the features assigned to energy transfer between the $T_{2 s}$ and $P_{2}$ modes that were visible for ssDNA when $n \geq 6$.[15] This peak (indicated by blue arrows in Figure $6(\mathrm{e}-\mathrm{f})$ ) was visible at $353 \mathrm{~K}$, indicating that it was not dependent upon the dsDNA conformation. However, the absence of this off-diagonal peak in the spectra of oligomers with $n \leq 4$ indicates that a minimum oligomer length is needed to facilitate energy transfer between the two vibrational modes. It was postulated previously that this peak may arise from local structure formation of the single oligomer[15] and the chain length dependence reported here would seem to add weight to that conclusion.

There is also a remarkable drop in the amplitude of any off-diagonal features from the $T_{2 s}$ base mode in the oligomers with $n \leq 4$. It is notable that, in the IR absorption spectra for these shorter oligomers, the $T_{2 S}$ mode is clearly the smallest absorption band compared to the others in this spectral region. This feature is also broader in the spectra for oligomers with $n \leq 4$. The lack of off-diagonal features linking this particular base mode with the backbone modes could be a result of reduced transition dipole strength in these shorter oligomers due to a lack of vibrational coupling between the transition dipoles as observed in $\alpha$-helical peptides.[33] Another possibility could be that in the shorter oligomers, energy from the $T_{25}$ mode is preferentially dissipated via interaction with the solvent environment in proximity with this $\mathrm{C}=\mathrm{O}$ group. The broadening of features in the FTIR spectra is indicative of increased interaction with the solvent and this direct base-water interaction may prove to be a more efficient pathway for vibrational relaxation than observed in the longer oligomers where some degree of hydrophobic collapse of the oligomer may be impossible.

Across the range of modes for which dynamics have been measured in this data, it is clear that no significant variation in timescales between the different lengths of DNA oligomers used in this study exists. In general, the spread in the dynamics throughout the oligomers, represented by error bars in Figure 8 was generally small and therefore the use average timescales as presented is a reasonable representation of the data.

The dynamics of the energy transfer behaviour of the off-diagonal features falls into two groups. The ribose modes $\left(\operatorname{Rib}_{1,2}\right)$ show faster dynamics and appear to act as an initial conduit for energy as the base modes relax. By contrast, the $P_{2}$ and $L_{2}$ modes show slower energy transfer dynamics, with the decaying signals lasting significantly longer than 5 ps. This is indicative of general pathway for energy transport in DNA oligomers that support the conclusions made previously.[15] Population relaxation from the bases to the ribose modes facilitates vibrational transfer to and then between the coupled backbone modes followed by subsequent dissipation of energy to the solvent environment via vibrations of the DNA backbone. It is also clear however that the energy transfer mechanism is complex and it is not possible to rule out the 
involvement of alternative pathways utilising low frequency vibrational modes.[30,34] In particular, the longtime duration of the signals suggest that the low frequency modes of the DNA oligomer may be coupled to the $P_{2}$ and $L_{2}$ modes of the backbone.[30,31,35] This is consistent with the established position of the phosphate group as the primary contact between DNA and the solvent.

For all the oligomers measured in this study, off-diagonal features in the backbone region 2D-IR spectra linking the base modes with the $L_{2}$, and $\mathrm{Rib}_{2}$ modes are unaffected by heating of the sample. The primary mechanism giving rise to these peaks has been identified as being energy transfer and the indication is that this process is insensitive to oligomer conformation and is active in both longer oligomers and single nucleotide building blocks. Therefore energy relaxation process of DNA would appear to be a property of the nucleotide rather than the secondary structure. This is consistent with the need for fast energy dissipation mechanisms to avoid the effects of photo-induced damage to exist in all oligomers, irrespective of base composition.

\section{Conclusions}

One and two-color 2D-IR spectroscopy has been used to investigate the coupling and vibrational energy relaxation mechanisms in a series of AT-rich DNA oligomers varying in length from one to 15 bases. The ability of the longer $(n \geq 6)$ oligomers to form a stable base paired geometry plays a key role in facilitating coupling between the base and phosphate backbone vibrational modes. Upon melting of these oligomers, 2D-IR offdiagonal features connecting the majority of the base modes to the $\mathrm{P}_{2}$ symmetric $\mathrm{PO}_{2}{ }^{-}$stretching mode were lost, indicating they are unique to ds-DNA. Varying the oligomer length of the DNA oligomers had no significant impact on the timescales of the vibrational energy relaxation process observed in time-resolved data. A previously proposed mechanism for a 15mer AT-DNA oligomer, where population relaxation of the base modes to lower frequency backbone modes, including those on the ribose unit, which are coupled to and thus allow energy transfer to the backbone is supported by the dynamic data presented in this study. It appears that the ability of DNA to effectively dissipate excess energy to prevent damage has roots in the mononucleotide building blocks of DNA, while the ds-DNA architecture gives rise to helix-specific vibrational interactions across the macromolecular structure.

\section{Acknowledgements}

The authors acknowledge funding from the STFC (ST/M000125/1 and NTH Programme Access funding), BBSRC (BB/L014335/1) and EPSRC (University of Strathclyde Doctoral training grant), which supported this work. 


\section{References}

[1] V. Kozich, L. Szyc, E.T.J. Nibbering, W. Werncke, T. Elsaesser, Ultrafast redistribution of vibrational energy after excitation of NH stretching modes in DNA oligomers, Chem. Phys. Lett. 473 (2009) 171175. doi:10.1016/j.cplett.2009.03.059.

[2] C.S. Peng, K.C. Jones, A. Tokmakoff, Anharmonic vibrational modes of nucleic acid bases revealed by 2D IR spectroscopy., J. Am. Chem. Soc. 133 (2011) 15650-15660. doi:10.1021/ja205636h.

[3] C. Lee, K.-H. Park, M. Cho, Vibrational dynamics of DNA. I. Vibrational basis modes and couplings., J. Chem. Phys. 125 (2006) 114508. doi:10.1063/1.2213257.

[4] C. Lee, M. Cho, Vibrational dynamics of DNA. II. Deuterium exchange effects and simulated IR absorption spectra., J. Chem. Phys. 125 (2006) 114509. doi:10.1063/1.2213258.

[5] C. Lee, K.-H. Park, J.-A. Kim, S. Hahn, M. Cho, Vibrational dynamics of DNA. III. Molecular dynamics simulations of DNA in water and theoretical calculations of the two-dimensional vibrational spectra., J. Chem. Phys. 125 (2006) 114510. doi:10.1063/1.2213259.

[6] C. Lee, M. Cho, Vibrational dynamics of DNA: IV. Vibrational spectroscopic characteristics of A-, B-, and Z-form DNA's., J. Chem. Phys. 126 (2007) 145102. doi:10.1063/1.2715602.

[7] A.T. Krummel, P. Mukherjee, M.T. Zanni, Inter and Intrastrand Vibrational Coupling in DNA Studied with Heterodyned 2D-IR Spectroscopy, J. Phys. Chem. B. 107 (2003) 9165-9169. doi:10.1021/jp035473h.

[8] A.T. Krummel, M.T. Zanni, DNA vibrational coupling revealed with two-dimensional infrared spectroscopy: insight into why vibrational spectroscopy is sensitive to DNA structure., J. Phys. Chem. B. 110 (2006) 13991-14000. doi:10.1021/jp062597w.

[9] L.A.I. Ramakers, G. Hithell, J.J. May, G.M. Greetham, P.M. Donaldson, M. Towrie, A.W. Parker, G.A. Burley, N.T. Hunt, Optimised DNA Minor Groove Binding Follows an Induced Fit Model, J. Phys. Chem. B. 121 (2017) 1295-1303.

[10] P.J. Sanstead, P. Stevenson, A. Tokmakoff, Sequence-Dependent Mechanism of DNA Oligonucleotide Dehybridization Resolved through Infrared Spectroscopy, J. Am. Chem. Soc. 138 (2016) 1179211801. doi:10.1021/jacs.6b05854.

[11] T. Siebert, B. Guchhait, Y. Liu, R. Costard, T. Elsaesser, Anharmonic Backbone Vibrations in Ultrafast Processes at the DNA-Water Interface, J. Phys. Chem. B. 119 (2015) 9670-9677. doi:10.1021/acs.jpcb.5b04499.

[12] B.P. Fingerhut, R. Costard, T. Elsaesser, Predominance of short range Coulomb forces in phosphatewater interactions-a theoretical analysis, J. Chem. Phys. 145 (2016) 115101. 
doi:10.1063/1.4962755.

[13] M. González-jiménez, G. Ramakrishnan, T. Harwood, A. Lapthorn, S. Kelly, E. Ellis, K. Wynne, Observation of coherent delocalised phonon-like modes in DNA under physiological conditions, Nat. Commun. 7 (2016) 1-8. doi:10.1038/ncomms11799.

[14] G. Hithell, M. González-jiménez, G.M. Greetham, P.M. Donaldson, M. Towrie, A.W. Parker, G.A. Burley, N.T. Hunt, Ultrafast 2D-IR and Optical Kerr Effect Spectroscopy Reveal the Impact of Duplex Melting on the Structural Dynamics of DNA, Phys. Chem. Chem. Phys. 19 (2017) 10333-10342. doi:10.1039/C7CP00054E.

[15] G. Hithell, D.J. Shaw, P.M. Donaldson, G.M. Greetham, M. Towrie, G.A. Burley, A.W. Parker, N.T. Hunt, Long-Range Vibrational Dynamics Are Directed by Watson-Crick Base Pairing in Duplex DNA, J. Phys. Chem. B. 120 (2016) 4009-4018. doi:10.1021/acs.jpcb.6b02112.

[16] M.K. Kuimova, J. Dyer, M.W. George, D.C. Grills, J.M. Kelly, P. Matousek, A.W. Parker, X.Z. Sun, M. Towrie, A.M. Whelan, Monitoring the effect of ultrafast deactivation of the electronic excited states of DNA bases and polynucleotides following $267 \mathrm{~nm}$ laser excitation using picosecond time-resolved infrared spectroscopy, Chem. Commun. (2005) 1182-1184. doi:10.1039/b414450c.

[17] M.K. Kuimova, A.J. Cowan, P. Matousek, A.W. Parker, X.Z. Sun, M. Towrie, M.W. George, Monitoring the direct and indirect damage of DNA bases and polynucleotides by using time-resolved infrared spectroscopy., Proc. Natl. Acad. Sci. U. S. A. 103 (2006) 2150-2153. doi:10.1073/pnas.0506860103.

[18] M. Towrie, G.W. Doorley, M.W. George, A.W. Parker, S.J. Quinn, J.M. Kelly, ps-TRIR covers all the bases - recent advances in the use of transient IR for the detection of short-lived species in nucleic acids, Analyst. 134 (2009) 1265-1273. doi:10.1039/b902108f.

[19] C.T. Middleton, K. de La Harpe, C. Su, Y.K. Law, C.E. Crespo-Hernández, B. Kohler, DNA Excited-State Dynamics: From Single Bases to the Double Helix, Annu. Rev. Phys. Chem. 60 (2009) 217-239. doi:10.1146/annurev.physchem.59.032607.093719.

[20] W.J. Schreier, T.E. Schrader, F.O. Koller, P. Gilch, C.E. Crespo-Hernandez, V.N. Swaminathan, T. Carell, W. Zinth, B. Kohler, Thymine Dimerization in DNA Is an Ultrafast Photoreaction, Science (80-. ). 315 (2007) 625-629. doi:10.1126/science.1135428.

[21] S. Perun, A.L. Sobolewski, W. Domcke, Ab initio studies on the radiationless decay mechanisms of the lowest excited singlet states of 9H-adenine, J. Am. Chem. Soc. 127 (2005) 6257-6265. doi:10.1021/ja044321c.

[22] H. Kang, K.T. Lee, B. Jung, Y.J. Ko, S.K. Kim, H. Kang, K.T. Lee, B. Jung, Y.J. Ko, S.K. Kim, Intrinsic Lifetimes of the Excited State of DNA and RNA Bases Intrinsic Lifetimes of the Excited State of DNA and RNA Bases, J. Am. Chem. Soc. 124 (2002) 12958-12959. doi:10.1021/ja027627x. 
[23] J.-M.L. Pecourt, J. Peon, B. Kohler, Ultrafast Internal Conversion of Electronically Excited RNA and DNA Nucleosides in Water [ J. Am. Chem. Soc. 2000 , 122 , 9348-9349]., J. Am. Chem. Soc. 123 (2001) 5166-5166. doi:10.1021/ja016042k.

[24] J.M.L. Pecourt, J. Peon, B. Kohler, DNA excited-state dynamics: Ultrafast internal conversion and vibrational cooling in a series of nucleosides, J. Am. Chem. Soc. 123 (2001) 10370-10378. doi:10.1021/ja0161453.

[25] G.W. Doorley, M. Wojdyla, G.W. Watson, M. Towrie, A.W. Parker, J.M. Kelly, S.J. Quinn, Tracking DNA excited states by picosecond-time-resolved infrared spectroscopy: Signature band for a chargetransfer excited state in stacked adenine-thymine systems, J. Phys. Chem. Lett. 4 (2013) 2739-2744. doi:10.1021/jz401258n.

[26] L.P. Deflores, R. a Nicodemus, A. Tokmakoff, Two-dimensional Fourier transform spectroscopy in the pump-probe geometry, Opt. Lett. 32 (2007) 2966-2968. doi:10.1364/OL.32.002966.

[27] S.-H. Shim, D.B. Strasfeld, Y.L. Ling, M.T. Zanni, Automated 2D IR spectroscopy using a mid-IR pulse shaper and application of this technology to the human islet amyloid polypeptide., Proc. Natl. Acad. Sci. U. S. A. 104 (2007) 14197-14202. doi:10.1073/pnas.0700804104.

[28] G.M. Greetham, P.M. Donaldson, C. Nation, I. V. Sazanovich, I.P. Clark, D.J. Shaw, A.W. Parker, M. Towrie, A $100 \mathrm{kHz}$ time-resolved multiple-probe femtosecond to second infrared absorption spectrometer, Appl. Spectrosc. 70 (2016) 645-653. doi:10.1177/0003702816631302.

[29] M. Banyay, M. Sarkar, A. Graslund, A library of IR bands of nucleic acids in solution, Biophys. Chem. 104 (2003) 477-488. doi:10.1016/S0301-4622.

[30] D. V Kurochkin, S.R.G. Naraharisetty, I. V Rubtsov, A relaxation-assisted 2D IR spectroscopy method, Proc. Natl. Acad. Sci. USA. 104 (2007) 14209-14214.

[31] S.R.G. Naraharisetty, V.M. Kasyanenko, I. V. Rubtsov, Bond connectivity measured via relaxationassisted two-dimensional infrared spectroscopy, J. Chem. Phys. 128 (2008) 104502. doi:10.1063/1.2842071.

[32] N.C. Seeman, An overview of structural DNA nanotechnology., Mol. Biotechnol. 37 (2007) 246-257. doi:10.1007/s12033-007-0059-4.

[33] P. Hamm, M.T. Zanni, Concepts and Methods of 2D Infrared Spectroscopy, Cambridge University Press: Cambridge, 2011.

[34] A.A. Kurnosov, I. V. Rubtsov, A.L. Burin, Communication: Fast transport and relaxation of vibrational energy in polymer chains, J. Chem. Phys. 142 (2015). doi:10.1063/1.4905076.

[35] I. V. Rubtsov, Relaxation-assisted two-dimensional infrared (RA 2DIR) method: accessing distances 
over 10 å and measuring bond connectivity patterns, Acc. Chem. Res. 42 (2009) 1385-1394. doi:10.1021/ar900008p. 